\title{
IS BRAIN-DERIVED NEUROTROPHIC FACTOR (BDNF) VAL66MET POLYMORPHISM ASSOCIATED WITH OBSESSIVE-COMPULSIVE DISORDER? A META-ANALYSIS
}

\author{
Shengdong Wang ${ }^{1,2}$, Xiaohong $\mathrm{Xu}^{2,3}$, Pan Yan ${ }^{1,2}$, Mingfeng Song ${ }^{1,2}$, Jing $\mathrm{Li}^{1,2}$ \& Shuqi Wang ${ }^{1,2}$ \\ ${ }^{I}$ The Molecular Biology Laboratory, Hangzhou Seventh People's Hospital, Hangzhou, China \\ ${ }^{2}$ Mental Health Center, Zhejiang University School of Medicine, Hangzhou, China \\ ${ }^{3}$ Department of Geriatric Psychiatry, Hangzhou Seventh People's Hospital, Hangzhou, China
}

received: 9.5.2019;

revised: 11.6.2019;

accepted: 18.6 .2019

\section{SUMMARY}

Background: Brain-derived neurotrophic factor (BDNF) polymorphism plays an important role in neural survival and was proposed to be related to obsessive-compulsive disorder (OCD). Genetic association studies of the BDNF Val66Met polymorphism (rs6265) in OCD have produced inconsistent results. A meta-analysis of studies was conducted to compare the frequency of the $B D N F$ Val66Met variant between cases with OCD and age-matched controls.

Subjects and methods: Electronic databases were searched for eligible articles in English and ten studies on the association of the BDNF Val66Met polymorphism with OCD were analysed.

Results: A total of ten studies involving 2306 cases with $O C D$ and 4968 healthy controls were included. Findings indicated that the BDNF Val66Met polymorphism was not associated with OCD. But there was a marginally significant effect of the BDNF Val66Met variant on $O C D$ in different ethnicity.

Conclusion: Findings from this meta-analytic investigation of published literature provide little support for the Val66Met variant of BDNF as a predictor of OCD. Future well-powered agnostic genome-wide association studies with more refined phenotype are needed to clarify genetic influences on $O C D$.

Key words: BDNF - Val66Met - obsessive-compulsive disorder - OCD - meta-analysis

$* * * * *$

\section{INTRODUCTION}

Obsessive-compulsive disorder (OCD) is a chronic psychiatric disorder mainly manifested by obsessive thoughts and compulsive actions, with a lifetime prevalence of approximately 1.1-3.3\% (Liu et al. 2015). However, this figure is tending to increase with better methods of diagnosis and as standards of living improve. There is evidence that OCD is mediated by specific neurobiologic dysfunctions with a genetic component and even though several systems of neurotransmission like the serotoninergic, dopaminergic and catecholaminergic systems have been analyzed through association studies; however, the findings remain inconsistent.

A single nucleotide polymorphism (rs6265), the substitution of valine/methionine at the 66 codon in the 5 pro-region of the human BDNF protein (Val66Met), has been found to obstruct the processing of the proBDNF polypeptide and thereby decrease the release of BDNF (Wang et al. 2015b). Many studies have shown that the BDNF valine-to-methonine substitution at codon 66 produces functional differences (Gattere et al. 2018, Notaras et al. 2015, Taj et al. 2018, Wang et al. 2015a, Zai et al. 2015). The Met allele is associated with decreased activity of the BDNF system (Rybakowski 2008). Mice with the BDNFMet/Met genotype showed almost a $30 \%$ reduction in regulated release of
BDNF. BDNFMet/Met mice also exhibit increased anxiety related behaviors (Chen et al. 2006) and reduced extinction of fear learning compared with BDNFVal/ Val mice (Soliman et al. 2010).

Association studies examining the role of the BDNF Val66Met polymorphism and OCD have been inconsistent, potentially due to low power in individual studies, etiological heterogeneity, or because of random error in the absence of a true effect (Lau \& Eley 2010). In this study, we carefully reviewed and selected qualified studies, using a meta-analysis to investigate the role of the BDNF Val66Met polymorphism in patients with OCD.

\section{SUBJECTS AND METHODS}

\section{Search Strategy}

All published studies examining the association of the BDNF Val66Met polymorphism with OCD were carefully selected by two independent authors. Data were collected from the electronic databases PubMed, Cochrane, Springer Link and ScienceDirect. These databases were searched from the first date available up to November 30, 2018. The keywords "BDNF", "brain-derived neurotrophic factor," "Val66Met," “rs6265," “G196A," “196G/A," “OCD,” “obsessive compulsive disorder" "obsessive compulsive symptoms," 
were used for searching. Meanwhile, references cited in all identified articles, and references cited in relevant review articles, were also examined. We only included data from full-length papers, not abstracts from conference proceedings or letters.

\section{Inclusion Criteria}

All case-control studies reporting genotype frequencies of the Val66Met polymorphisms in OCD and in psychiatrically healthy controls were eligible for inclusion in this meta-analysis. The diagnosis of OCD was based on either the Diagnostic and Statistical Manual of Mental Disorders (DSM) or International Classification of Diseases (ICD), including DSM-III, DSM-III-R, DSM-IV, DSM-IV-R, and ICD-10. We included studies involving samples with any ethnic background. Case-only studies, family-based designs, and population-based studies of healthy subjects were not included.

\section{Data Extraction}

Two authors independently extracted data to avoid potential mistakes. Discrepancies were resolved by discussions within the research team. From each study, we extracted the first author's name, year of publication, ethnicity of samples, diagnostic criteria of OCD, number of cases and controls, and the available genotype information of the BDNF Val66Met polymorphism.

\section{Meta-Analysis Methods}

We examined the relationship between the frequency of the Met allele and diagnosis of OCD. The odds ratio (OR) and its $95 \%$ confidence interval $(95 \%$ CI) were estimated for each study. We performed a chi-squared-based Q-statistic test to assess betweenstudy heterogeneity (Lau et al. 1997). In this analysis, $\mathrm{P}<0.1$ indicates the presence of significant heterogeneity. The inconsistency index $\mathrm{I}^{2}$ was also calculated to evaluate heterogeneity. Depending on the results of the heterogeneity tests among individual studies, we used a fixed-effects model (if $\mathrm{P}>0.1$ ) (Mantel \& Haenszel 1959) or a random-effects model (if $\mathrm{P}<0.1$ ) (DerSimonian \& Laird 1986) to summarize the pooled OR. We used a Z-test with a significance threshold of $\mathrm{P}<0.05$ to evaluate the OR.

\section{Evaluation of Publication Bias}

Publication bias, that is, the preferential publication of a study with positive findings, was evaluated with a funnel plot such that asymmetry of the funnel plot suggests bias. All analyses were performed using the software Stata (version 14.0, Stata Corp LP, College Station, TX).

\section{RESULTS}

\section{Description of Studies Identified in Meta-Analysis}

We identified 45 potentially relevant research papers using our search strategies, but 35 did not meet the inclusion criteria (Figure 1) after reviewing the abstracts or full text. Finally, Ten case-control studies (da Rocha 2010, Hemmings et al. 2008, Katerberg et al. 2009, Liu et al. 2015, Marquez et al. 2013, Taj et al. 2018, Tukel et al. 2012, Umehara et al. 2016, Wang et al. 2015b, Wendland et al. 2007) with a total of 2306 OCD patients and 4968 psychiatrically healthy controls were included in the meta-analysis. As one study (Katerberg et al. 2009) contained separate data of two different groups of subjects, we treated them independently, therefore we obtained eleven eligible samples. The quality of the included studies was adequate, with a range from four to nine stars and a mean value of $7.4 \pm 1.4$ stars (Table 1). The flow diagram of the study selection is depicted in Figure 1. The detailed characteristics of the studies included are presented in table 2 .

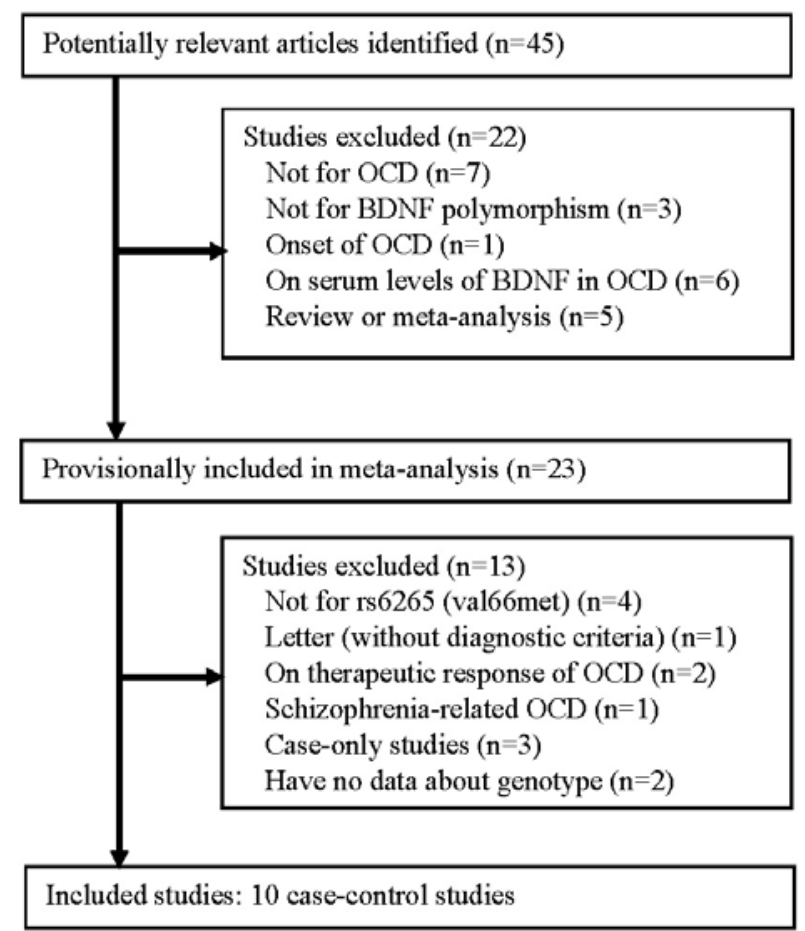

Figure 1. The flow diagram of the study selection

\section{Effect of BDNF Val66Met on OCD}

All data were pooled together (ten studies with a total of 2306 OCD patients and 4968 psychiatrically healthy controls), significant differences in heterogeneity were observed in the allelic model $\left(\mathrm{I}^{2}=77.6 \%, \mathrm{p}<0.001\right)$, the additive model $\left(\mathrm{I}^{2}=62.4 \%, \mathrm{p}=0.003\right)$, the dominant model $\left(\mathrm{I}^{2}=71.2 \%, \mathrm{p}<0.001\right)$ and the recessive model $\left(\mathrm{I}^{2}=56.2 \%\right.$, $\mathrm{p}=0.011)$. Therefore, the random effects model was chosen to calculate the pooled OR and $95 \%$ CI. Results 
Table 1. Quality ratings for studies included on the basis of Newcastle-Ottawa quality assessment scale

$$
\text { Selection Comp. Exposure }
$$

Study

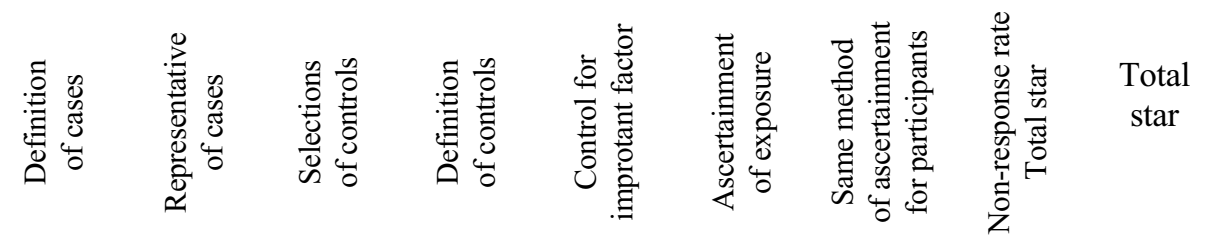

Wendland et al.2007

Hemmings et al. 2008

Katerberg et al.2009 I

Katerberg et al.2009 II

Rocha et al. 2010

Tukel et al. 2012

Marquez et al. 2013

Liu et al. 2015

Wang et al. 2015

Umehara et al. 2016

Tai et al. 2017

Average star (means \pm SD)

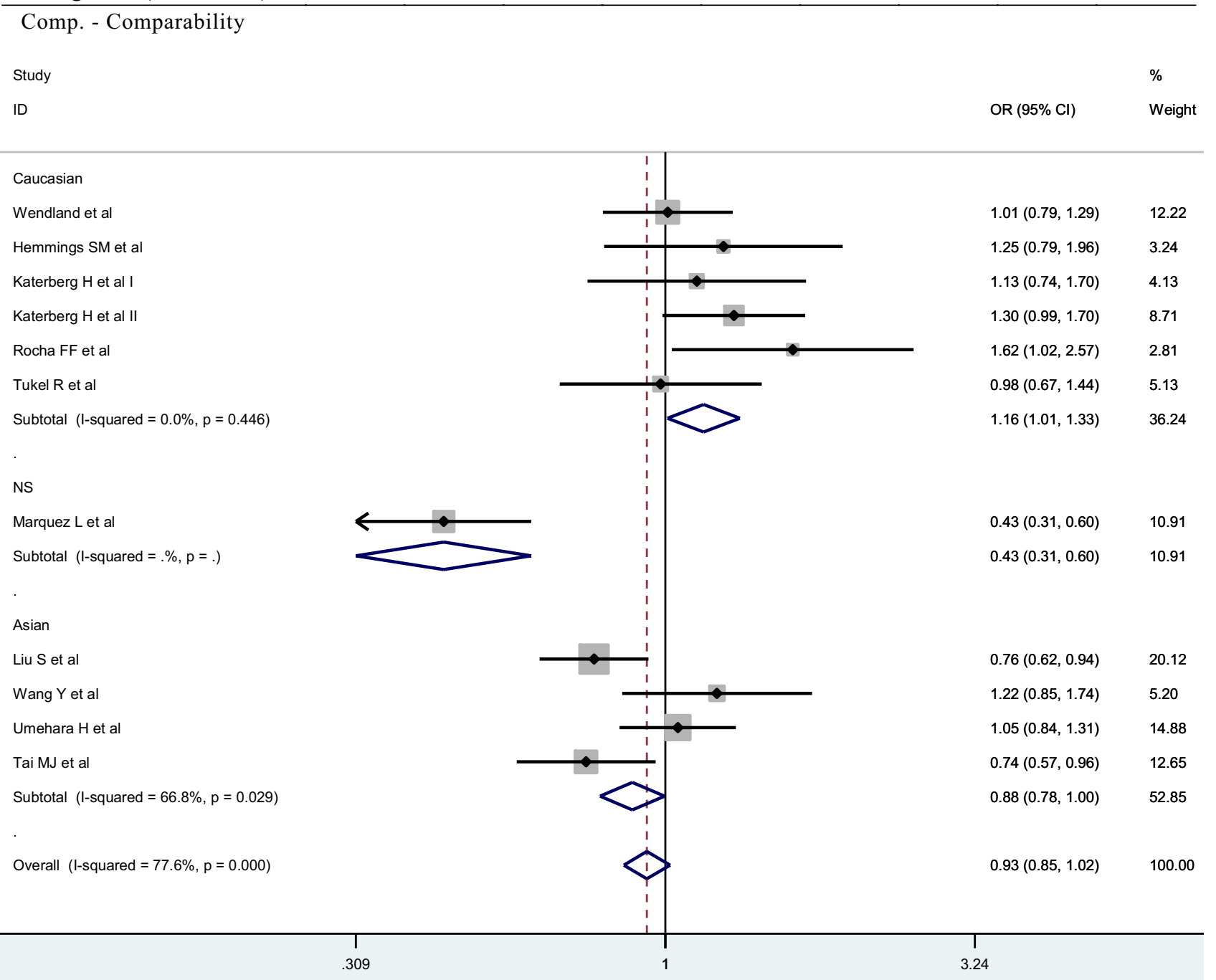

Figure 2. Forest plot for Met versus Val (allelic model) in total or subgroup 

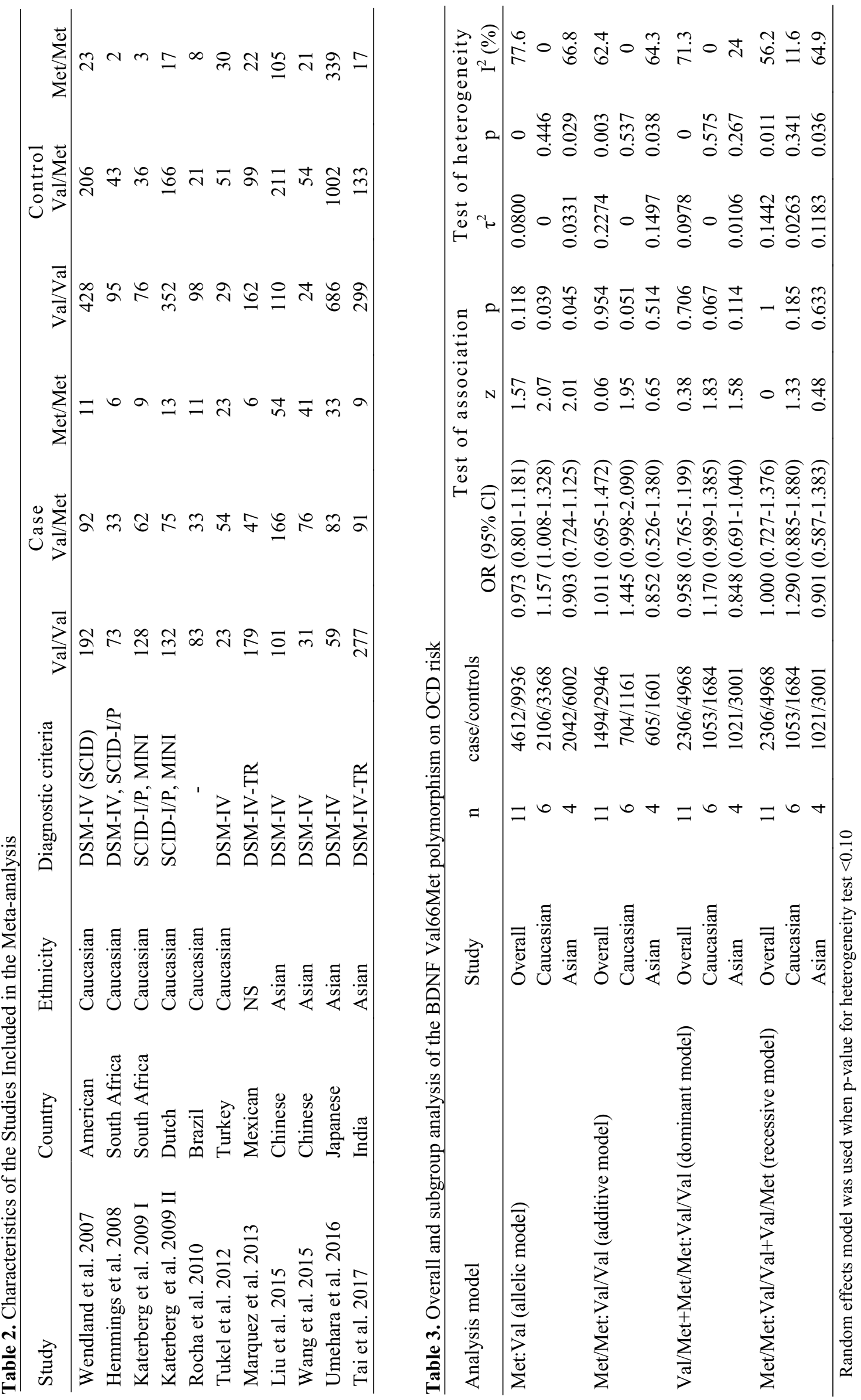


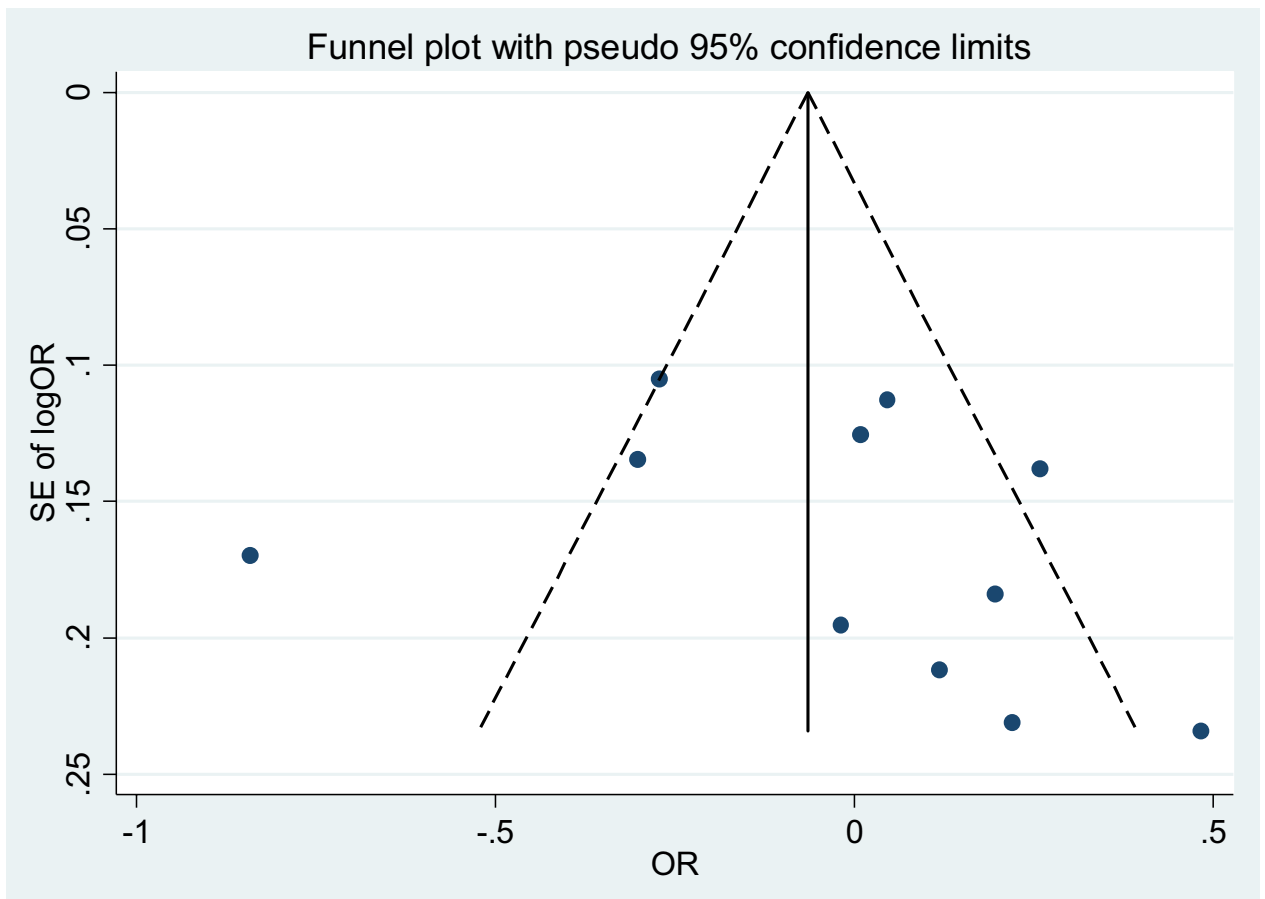

Figure 3. Funnel plot of Met versus Val (allelic model) in total. OR, odds ratio

indicated no significant association between BDNF Val66Met polymorphism and OCD in all four models above ( $>0.05$ for each comparison, table 3 ). The between-study heterogeneity in the pooled results was obvious and previous studies have found significant ethnic difference of the BDNF Val66Met polymorphism frequencies, thus we performed subgroup analyses by ethnicity. Results indicate a significant effect for the Met allele on the risk of OCD in Caucasian (OR 1.157, CI 1.008-1.328, $\mathrm{p}=0.039$ ), and a significant effect for the Val allele on the risk of OCD in Asian (OR 0.903, CI 0.724-1.125, $\mathrm{p}=0.045$ ). Forest plots depicting effect sizes and their CIs for the included studies can be seen in Figure 2.

\section{Publication Bias}

A funnel plot was performed to assess the potential publication bias. The shape of the funnel plot was not so asymmetrical when comparing the Met allele with the Val allele (Figure 3), which indicated that a potential publication bias may be existed. We further evaluated it by Egger's test, whereas it showed no publication bias (coefficient $=2.21 ; 95 \%$ CI: $-3.27-7.69 ; \mathrm{p}=0.386>0.05$ ).

\section{DISCUSSION}

Previous studies have indicated that the Val66Met polymorphism of BDNF gene might play an important role in the pathophysiology of several psychotic disorders (Gattere et al. 2018, Kirli et al. 2018, Rybakowski 2008). The association between Val66Met and OCD susceptibility has been widely studied, although inconsistent results were obtained. Several studies performed the comprehensive meta-analysis of the genetic association studies of OCD susceptibility, and found there were no significant association between Val66Met and OCD (Taylor 2013; Wang et al. 2015a; Zai et al. 2015). In the present meta-analysis, we retrieved a total of 2306 OCD patients and 4968 psychiatrically healthy controls through a structured literature search and performed the analysis for four genetic models, but also failed to find an association between BDNF Val66Met polymorphism and OCD in any comparison. This result is consistent with previous findings.

Some studies have found significant ethnic differences of the BDNF Val66Met polymorphism frequencies (Shimizu et al. 2004). In order to clarify whether OCD susceptibility is correlated with the ethnic differences of the Val66Met polymorphism, we performed subgroup analyses by ethnicity. Results indicate a significant effect for the Met allele on the risk of OCD in Caucasian (OR 1.157, CI 1.008-1.328, $\mathrm{p}=0.039$ ), and a significant effect for the Val allele on the risk of OCD in Asian (OR 0.903, CI 0.724-1.125, p=0.045). The present updated meta-analysis was inconsistent with previous study (Wang et al. 2015a).

Genetic association studies have shown that the Val66Met polymorphism may be a risk factor for the development of neuropsychiatric disorders (Hong et al., 2011), and that there is evidence that the polymorphism modulates aspects of neurocognitive function in otherwise healthy adults (Dincheva et al. 2012). Since 2003, the year in which the Val66Met polymorphism was first described as a functional variant (Egan et al. 2003), the number of studies evaluating the effects of this evolutionarily recent genetic aberration has progressively increased, especially in psychiatric disorders. The evidence suggesting that the 66Met allele is associated with risk of 
developing schizophrenia is relatively weak (Zakharyan et al. 2011), with most studies reporting no evidence that the Val66Met polymorphism is significantly overrepresented among schizophrenia patients relative to controls. But there have been several positive reports that the $66 \mathrm{Met}$ allele may be related to age of onset of schizophrenia (Zakharyan et al. 2011, Numata et al. 2006, Chao et al. 2008, Decoster et al. 2011). Therapeutic efficacy also appears to be gated by the Val66Met polymorphism (Zai et al. 2012, Hong et al. 2003). The Val66Met polymorphism has also been linked to the presence of depressive symptoms in schizophrenia (Schumacher et al. 2005). In summary, the collective Val66Met polymorphism literature suggests a complex and multifaceted role of BDNF function in psychiatric disorder susceptibility. Whether BDNF is a viable therapeutic target for the treatment of psychiatric disorders remains a contentious topic, yet continued study of the Val66Met polymorphism holds value by providing insight into the effects of altered activity-dependent BDNF signaling on brain structure, function and disorder.

There are some limitations in the present meta-analysis. First, the number of available case - control studies on BDNF Val66Met polymorphism and OCD is relatively small. Besides, there may be genetic difference between early-onset and late-onset OCD (Katerberg et al. 2009), but we did not perform a stratified analysis based upon the onset age owing to the unavailability of the necessary data. Moreover, despite that we strived to strict the inclusion and exclusion criteria when retrieving eligible studies, a potential publication bias could still exist owing to a relative small number of studies included; and it may threaten the validity of conclusions. Given these reasons, the interpretation of the results should be cautious, although the overall quality of the included studies was adequate. OCD and the search for candidate genes have been the topic of numerous studies. Findings have been inconsistent, which may be due to varying samples and methodology. It is important to note that a heterogeneous disorder such as OCD cannot fully be explained by a simple one-gene cause and effect model. This lends support to not only a polygenic model but also to the possible role of environment-gene interactions.

\section{CONCLUSION}

In conclusion, this study indicates that the allele Met is the risk of OCD in Caucasian, but is a protector of OCD in Asian. The exact biological mechanism of this funding needs further study.

\section{Acknowledgements:}

This study was supported by Zhejiang Provincial Natural Science Foundation of China (LY17H090013)

Conflict of interest: None to declare.

\section{Contribution of individual authors:}

Shengdong Wang \& Xiaohong Xu were responsible for study design and responsible for manuscript;

Xiaohong $\mathrm{Xu}$, \& Jing Li were responsible for literature review and data collection;

Shengdong Wang, Pan Yan, Mingfeng Song \& Shuqi Wang were responsible for statistical analysis.

\section{References}

1. Chao HM, Kao HT, Porton B: BDNF Val66Met variant and age of onset in schizophrenia. Am J Med Genet B 2008; 147:505-506

2. Chen ZY, Jing D, Bath KG, Ieraci A, Khan T, Siao CJ, Herrera DG, Toth M, Yang C, McEwen BS, Hempstead BL, Lee FS: Genetic variant BDNF (Val66Met) polymorphism alters anxiety-related behavior. Science 2006; 314:140-143

3. Decoster J, van Os J, Kenis G, Henquet C, Peuskens J, De Hert $M$, van Winkel R: Age at onset of psychotic disorder: cannabis, BDNF Val66Met, and sex-specific models of geneenvironment interaction. Am J Med Genet B 2011; 156:363-369

4. DerSimonian R, Laird N: Meta-analysis in clinical trials. Controlled clinical trials 1986; 7:177-188

5. Dincheva I, Glatt CE, Lee FS: Impact of the BDNF Val66Met polymorphism on cognition implications for behavioral genetics. Neuroscientist 2012; 18:439-451

6. Egan MF, Kojima M, Callicott JH, Goldberg TE, Kolachana BS, Bertolino A, Zaitsev E, Gold B, Goldman D, Dean $M, L u B$, Weinberger $D R$ : The BDNF val66met polymorphism affects activity-dependent secretion of $B D N F$ and human memory and hippocampal function. Cell 2003; 112:257-269

7. Gattere G, Stojanovic-Perez A, Monseny R, Martorell L, Ortega L, Montalvo I, Sole M, Algora MJ, Cabezas A, Reynolds RM, Vilella E, Labad J: Gene-environment interaction between the brain-derived neurotrophic factor Val66Met polymorphism, psychosocial stress and dietary intake in early psychosis. Early intervention in psychiatry, 2018; 12:811-820

8. Hemmings SM, Kinnear CJ, Van der Merwe L, Lochner C, Corfield VA, Moolman-Smook JC, Stein DJ: Investigating the role of the brain-derived neurotrophic factor (BDNF) val66met variant in obsessive-compulsive disorder (OCD). The world journal of biological psychiatry: the official journal of the World Federation of Societies of Biological Psychiatry 2008; 9:126-134

9. Hong CJ, Liou YJ, Tsai SJ: Effects of BDNF polymorphisms on brain function and behavior in health and disease. Brain research bulletin 2011; 86:287-297

10. Hong CJ, Yu YW, Lin CH, Tsai SJ: An association study of a brain-derived neurotrophic factor Val66Met polymorphism and clozapine response of schizophrenic patients. Neurosci Lett 2003; 349:206-208

11. Katerberg H, Lochner C, Cath DC, de Jonge P, Bochdanovits Z, Moolman-Smook JC, Hemmings SM, Carey PD, Stein DJ, Sondervan D, Boer JA, van Balkom AJ, Polman A, Heutink P: The role of the brain-derived neurotrophic factor (BDNF) val66met variant in the phenotypic expression of obsessive-compulsive disorder (OCD). American 
journal of medical genetics. Part B, Neuropsychiatric genetics. International Society of Psychiatric Genetics 2009; 150:1050-1062

12. Kirli U, Binbay T, Drukker M, Elbi H, Kayahan B, Gokcelli $D K$, Ozkinay F, Onay H, Alptekin K, van Os J: Is BDNFVal66Met polymorphism associated with psychotic experiences and psychotic disorder outcome? Evidence from a 6 years prospective population-based cohort study. American journal of medical genetics. Part B, Neuropsychiatric genetics. International Society of Psychiatric Genetics, 2018

13. Lau J, Ioannidis JP, Schmid CH: Quantitative synthesis in systematic reviews. Annals of internal medicine 1997; 127:820-826

14. Lau JY, Eley TC: The genetics of mood disorders. Annual review of clinical psychology 2010; 6:313-337

15. Liu S, Cui J, Niu Z, Yi M, Zhang X, Che F, Ma X: Do obsessive-compulsive disorder and Tourette syndrome share a common susceptibility gene? An association study of the $B D N F$ Val66Met polymorphism in the Chinese Han population. The world journal of biological psychiatry. 2015; 1-8

16. Mantel N, Haenszel W: Statistical aspects of the analysis of data from retrospective studies of disease. Journal of the National Cancer Institute 1959; 22:719-748

17. Marquez L, Camarena B, Hernandez S, Loyzaga C, Vargas $L$, Nicolini H: Association study between BDNF gene variants and Mexican patients with obsessive-compulsive disorder. European neuropsychopharmacology. European College of Neuropsychopharmacology 2013; 23:1600-1605

18. Notaras M, Hill R, van den Buuse M: The BDNF gene Val66Met polymorphism as a modifier of psychiatric disorder susceptibility: progress and controversy. Molecular psychiatry 2015; 20:916-930

19. Numata S, Ueno S, Iga J, Yamauchi K, Hongwei S, Ohta K, Kinouchi S, Shibuya-Tayoshi S, Tayoshi S, Aono M, Kameoka N, Sumitani S, Tomotake M, Kaneda Y, Taniguchi T, Ishimoto Y, Ohmori T: Brain-derived neurotrophic factor (BDNF) Val66Met polymorphism in schizophrenia is associated with age at onset and symptoms. Neurosci Lett 2006; 401:1-5

20. Rocha FF, Malloy-Diniz L, Lage NV, Correa H: Positive association between MET allele (BDNF Val66Met polymorphism) and obsessive compulsive disorder. Revista brasileira de psiquiatria 2010; 32:323-324

21. Rybakowski JK: BDNF gene: functional Val66Met polymorphism in mood disorders and schizophrenia. Pharmacogenomics 2008; 9:1589-1593

22. Schumacher J, Jamra RA, Becker T, Ohlraun S, Klopp N, Binder EB, Schulze TG, Deschner M, Schmal C, Hofels $S$, Zobel A, Illig T, Propping P, Holsboer F, Rietschel M, Nothen MM, Cichon S: Evidence for a relationship between genetic variants at the brain-derived neurotrophic factor (BDNF) locus and major depression. Biol Psychiatry 2005; 58:307-314

23. Shimizu E, Hashimoto K, Iyo M: Ethnic difference of the BDNF 196G/A (val66met) polymorphism frequencies: the possibility to explain ethnic mental traits. American journal of medical genetics. Part B, Neuropsychiatric genetics. International Society of Psychiatric Genetics 2004; 126:122-123

24. Soliman F, Glatt CE, Bath KG, Levita L, Jones RM, Pattwell SS, Jing D, Tottenham N, Amso D, Somerville LH, Voss HU, Glover G, Ballon DJ, Liston C, Teslovich T, Van Kempen T, Lee FS, Casey BJ: A genetic variant $B D N F$ polymorphism alters extinction learning in both mouse and human. Science 2010; 327:863-866

25. Taj MJR, Ganesh S, Shukla T, Deolankar S, Nadella RK, Sen S, Purushottam M, Reddy YCJ, Jain S, Viswanath B: $B D N F$ gene and obsessive compulsive disorder risk, symptom dimensions and treatment response. Asian $J$ Psychiatr 2018; 38:65-69

26. Taylor S: Molecular genetics of obsessive-compulsive disorder: a comprehensive meta-analysis of genetic association studies. Molecular psychiatry 2013; 18:799-805

27. Tukel R, Gurvit H, Ozata B, Ozturk N, Ertekin BA, Ertekin, E, Baran B, Kalem SA, Buyukgok D, Direskeneli GS: Brain-derived neurotrophic factor gene Val66Met polymorphism and cognitive function in obsessive-compulsive disorder. American journal of medical genetics. Part $B$, Neuropsychiatric genetics. International Society of Psychiatric Genetics 2012; 159:850-858

28. Umehara H, Numata S, Kinoshita $M$, Watanabe $S$, Nakaaki S, Sumitani S, Ohmori T: No association between BDNF Val66Met polymorphism and treatment response in obsessive-compulsive disorder in the Japanese population. Neuropsychiatric disease and treatment 2016; 12:611-615

29. Wang J, Zhang F, Zhu W, Liu Y, Zhou Z: Meta-analysis of the association of brain-derived neurotrophic factor Val66Met polymorphism with obsessive-compulsive disorder. Acta neuropsychiatrica 2015a; 27:327-335

30. Wang Y, Zhang H, Li Y, Wang Z, Fan Q, Yu S, Lin Z, Xiao Z: BDNF Val66Met polymorphism and plasma levels in Chinese Han population with obsessive-compulsive disorder and generalized anxiety disorder. J Affect Disord $2015 b ; 186: 7-12$

31. Wendland JR, Kruse MR, Cromer KR, Murphy DL: A large case-control study of common functional SLC6A4 and BDNF variants in obsessive-compulsive disorder. American College of Neuropsychopharmacology 2007; 32:2543-2551

32. Zai G, Zai CC, Arnold PD, Freeman N, Burroughs E, Kennedy JL, Richter MA: Meta-analysis and association of brain-derived neurotrophic factor (BDNF) gene with obsessive-compulsive disorder. Psychiatric genetics 2015; 25:95-96

33. Zai GC, Zai CC, Chowdhury NI, Tiwari AK, Souza RP, Lieberman JA, Meltzer HY, Potkin SG, Muller DJ, Kennedy JL: The role of brain-derived neurotrophic factor (BDNF) gene variants in antipsychotic response and antipsychotic-induced weight gain. Prog Neuropsychopharmacol Biol Psychiatry 2012; 39:96-101

34. Zakharyan R, Boyajyan A, Arakelyan A, Gevorgyan A, Mrazek F, Petrek M: Functional variants of the genes involved in neurodevelopment and susceptibility to schizophrenia in an Armenian population. Hum Immunol 2011; 72:746-748

Correspondence:

Shengdong Wang, MD

The Molecular Biology Laboratory, Hangzhou Seventh People's Hospital

Hangzhou 310013, China

E-mail:wangsd0108@sina.com 